\title{
Survival prognostic and recurrence risk factors after single pulmonary metastasectomy
}

\author{
Céline Forster ${ }^{1}$, Amaya Ojanguren ${ }^{1}$, Jean Yannis Perentes ${ }^{1,2}$, Matthieu Zellweger $^{1}$, Thorsten Krueger ${ }^{1,2}$, \\ Etienne Abdelnour-Berchtold ${ }^{1}$ and Michel Gonzalez ${ }^{1,2^{*}}$ (D)
}

\begin{abstract}
Background: Identification of the prognostic factors of recurrence and survival after single pulmonary metastasectomy (PM).

Methods: Retrospective analysis of all consecutive patients who underwent PM for a single lung metastasis between 2003 and 2018.

Results: A total of 162 patients with a median age of 64 years underwent single PM. Video-Assisted Thoracic Surgery (VATS) was performed in $83.9 \%$ of cases. Surgical resection was achieved by wedge in $73.5 \%$, segmentectomy in $7.4 \%$, lobectomy in $17.9 \%$ and pneumonectomy in $1.2 \%$ of cases. The median durations of hospital stay and of drainage were 4 days (IQR 3-7) and 1 day (IQR 1-2), respectively. During the follow-up (median 31 months; IQR 15-58), 93 patients (57.4\%) presented recurrences and repeated PM could be realized in 35 patients (21.6\%) achieved by VATS in $77.1 \%$. Non-colorectal tumour (HR 1.84), age < 70 years (HR 1.77) and previous extra-thoracic metastases (HR 1.61) were identified as prognostic factors of recurrence. Overall survival at 5 -year was estimated at $67 \%$. Non-colorectal tumour (HR 2.40) and mediastinal lymph nodes involvement (HR 3.42) were significantly associated with an increased risk of death.
\end{abstract}

Conclusions: Despite high recurrence rates after PM, surgical resection shows low morbidity rate and acceptable long-term survival, thus should remain the standard treatment for single pulmonary metastases.

Trial registration: The Local Ethics Committee approved the study (No. 2019-02,474) and individual consent was waived.

Keywords: Pulmonary metastases, Pulmonary metastasectomy, VATS

\section{Background}

Pulmonary metastases occur in $30-40 \%$ of patients with known solid cancer and their appearance indicates a progression of the primary tumour, thus a worsening of the prognosis [1]. Surgical resection of pulmonary metastases may be considered a valid part of the multimodal treatment in patients who have no other distant metastases

*Correspondence: michel.gonzalez@chuv.ch

1 Service of Thoracic Surgery, Lausanne University Hospital (CHUV), Rue du Bugnon 46, 1011 Lausanne, Switzerland

Full list of author information is available at the end of the article and can tolerate complete resection of the metastases [2]. In addition to local disease control, surgery allows confirmation of the diagnosis by providing suitable metastatic tissue for histopathological analysis. This facilitates more personalized subsequent systemic treatments, such as targeted therapy or immunotherapy [3].

Patients presenting only a single pulmonary metastasis represent $45-75 \%$ of all patients with pulmonary metastases $[4,5]$. Single pulmonary metastases are generally managed surgically with a curative intent, but recently there has been a growing interest in various non-surgical approaches, such as radiofrequency or stereotaxic 
radiotherapy, as promising alternatives in terms of local control [6]. Complete resection of solitary metastases is generally achieved by non-anatomical wedge resection when they are in peripheral locations. Anatomical resections (segmentectomy or lobectomy) may be an alternative for larger or central lesions. Minimally invasive approaches are gaining acceptance in pulmonary metastasis patients, leading to lower post-operative morbidity, shorter duration of hospital stay and oncological outcomes equivalent to those of open thoracotomy [7, 8]. Patients with single pulmonary metastases have been reported to present a better prognosis and an increased overall survival in comparison to patients bearing multiple pulmonary metastases [5, 9-11]. However, little is known about the recurrence rate and risk factors in this specific group of patients. Because they represent a nonnegligible sub-sample of all PM patients, and because the specific configuration of a single lung metastasis sets these patients apart in terms of possible surgical cure of their disease, we herewith propose to focus on them only.

The aim of this study was to analyze the surgical outcomes of patients operated for a single pulmonary metastasis. We also evaluated the risk factors for recurrence and identified prognostic factors of shorter survival.

\section{Methods}

\section{Patient selection and study design}

This retrospective study reviewed all consecutive patients with a single pulmonary metastasis who underwent a surgical pulmonary metastasectomy (PM) with curative intent in our institution between July 2003 and November 2018. Patients undergoing diagnostic procedures only and non-surgical patients were excluded. A total of 264 patients were treated in the period of interest, of which 162 had a single pulmonary metastasis. Pre-operatively, all patients were discussed by an interdisciplinary tumour board to assess the criteria, which had to be fulfilled for PM [2]. These are: (1) the patient can withstand surgical intervention; (2) the primary tumour is controlled; (3) there is no other extra-pulmonary metastases or if there is, it can be resected completely before PM; (4) the pulmonary metastasis can be completely resected without impacting the patient's respiratory functions; (5) there is no other alternative systemic treatment. All patients underwent a thin-slice $(1 \mathrm{~mm})$ helical chest CTscan within 30 days before surgery, which was reviewed by a senior radiologist. Moreover, a Positron Emission Tomography (PET) scan was realized when the primary tumour showed high uptake of Fluorodeoxy-Glucose (FDG) to exclude extra-thoracic metastases. Pre-operative diagnosis by bronchoscopy or percutaneous biopsy under CT-guidance was individually discussed but not mandatory.

\section{Data collection}

We retrospectively extracted the data from our electronic database and reviewed medical records. Data included: patient's demographics; comorbidities; primary tumour and pulmonary metastasis histology; surgical characteristics; post-operative outcomes up to 30 days after surgery; recurrences, if any, and their localisation; repeated PM characteristics. In addition, disease-free interval (DFI) and overall survival (OS) were assessed. The DFI1 was defined as the interval between the primary tumour resection and the first PM and the DFI2 was defined as the interval between first $\mathrm{PM}$ and cancer recurrence. OS was defined as the percentage of patients alive on November 2019, when the follow-up was completed or date of last news. Pulmonary metastases that were diagnosed at the same time as the primary tumour diagnosis were defined as synchronous metastases.

The Local Ethics Committee approved the study (No. 2019-02474) and individual consent was waived.

\section{Operation and follow-up}

The PM was performed under general anaesthesia with single-lung ventilation. If necessary, a percutaneous hook wire device was inserted under CT-scan guidance in the pre-operative phase to facilitate the intraoperative detection of the metastasis. The surgical approach (open or thoracoscopy) and the extent of pulmonary resection (wedge or anatomical resection) were individually discussed for all patients based on the metastasis characteristics (size, localisation). Parenchyma-sparing resection (wedge) and minimally-invasive approach were preferred when feasible. Our Video-Assisted Thoracic Surgery (VATS) approach consisted in a classical three-port anterior approach and the thoracotomy was a standard posterolateral incision in the fifth intercostal space. Lymph node involvement was not considered a contra-indication for surgery. However, lymph node dissection was only realized for lesions of more than $2 \mathrm{~cm}$ in diameter, centrally located, requiring an anatomical resection, or when lymph node involvement was suspected on pre-operative radiological exams. All specimens were extracted in a protective bag. The specimens were then examined by the operator in order to assess the completeness of resection. In case of doubt, a histological frozen section was performed. After surgery, all cases were discussed once again in the interdisciplinary tumour board to evaluate the indications for an adjuvant therapy. The follow-up consisted in a thoraco-abdominal CT-scan at 3, 6, 12, 18 and 24 months and then on a yearly basis. 


\section{Statistical analysis}

Binary variables are expressed as percentages and continuous variables are presented as median with interquartile range (IQR). OS and PFS were calculated using the Kaplan-Meier and log-rank analyses. Cox regression for uni- and multivariable analyses were applied to identify prognostic factors of recurrence and survival. A two-tailed hypothesis was used and significance accepted if $p<0.05$. The statistical analysis was performed using the Stata version 14 software (StataCorp, Texas USA).

\section{Results}

Single PM was performed in a total of 162 patients (female/male: 72/90). Patient characteristics are shown in Table 1 . The primary tumour origin included colorectal carcinoma (31.5\%), melanoma (20.4\%), sarcoma (14.8\%) and other origins (33.3\%). Pulmonary metastases were synchronous in $14.2 \%$ and metachronous in $85.8 \%$ of cases. In case of metachronous metastases, the median DFI1 was 22.5 months (IQR 10-47 months). Fifty patients $(30.9 \%)$ had been previously treated by radiotherapy or surgery for another metastases, with $36 \%$ of cases in the liver, $28 \%$ in local lymph nodes, $6 \%$ in the brain and $30 \%$ in other localisations. Chemotherapy was administered in 75 patients (46.3\%) before the first PM.

A VATS was performed in 136 patients (83.9\%) and open thoracotomy in 26 patients $(16.1 \%)$. There was one case of conversion (accounted for in the latter group) because of a centrally located lesion non-resectable by VATS. Surgical resection was achieved by wedge in $73.5 \%$, segmentectomy in $7.4 \%$, lobectomy in $17.9 \%$ and pneumonectomy in $1.2 \%$ of cases. Mediastinal lymph node dissection was performed in 36 patients (22.2\%). The median durations of drainage and of hospital stay were 1 day (IQR 1-2) and 4 days (IQR 3-7), respectively. The overall post-operative complication rate at 30 days was $11.7 \%$, with $7.4 \%$ of pulmonary and $1.9 \%$ of cardiac complications. During the 30 post-operative days (POD) period, two patients (1.2\%) were readmitted. The first one presented an inflammatory lung effusion which was treated by drainage. The second one had positive histopathological margins and the resection was therefore completed. There was no 30-day mortality.

The median follow-up time was 31.5 months (IQR 15-58). Recurrences were observed in 93 patients (57.4\%), with $13 \%$ in the lung only, $16.1 \%$ distantly and $28.4 \%$ in both localisations (Table 2). Lung recurrences were ipsilateral in $50.8 \%$ of cases, contralateral in $31.3 \%$ and bilateral in $17.9 \%$ of cases. The median DFI2 was 11 months (IQR 3-31). Thirty-five patients (21.6\%) underwent repeated PM (RPM), by VATS in $77.1 \%$ and
Table 1 Patient characteristics and surgical characteristics of first single pulmonary metastasectomy (PM)

\begin{tabular}{|c|c|}
\hline Single PM & 162 \\
\hline \multicolumn{2}{|l|}{ Sex } \\
\hline Female & $72(44.4 \%)$ \\
\hline Male & $90(55.6 \%)$ \\
\hline Age (median) & 64 [IQR 55-71] \\
\hline \multicolumn{2}{|l|}{ Comorbidities } \\
\hline Cardiopathy & $12(7.4 \%)$ \\
\hline High blood pressure & $61(37.7 \%)$ \\
\hline Pulmonary disease & $13(8 \%)$ \\
\hline Tobacco exposure & $49(30.3 \%)$ \\
\hline Diabetes & $23(14.2 \%)$ \\
\hline Renal failure & $11(6.8 \%)$ \\
\hline Immunosuppression & $6(3.7 \%)$ \\
\hline \multicolumn{2}{|l|}{ Primary tumour } \\
\hline Colorectal & $51(31.5 \%)$ \\
\hline Melanoma & $33(20.4 \%)$ \\
\hline Sarcoma & $24(14.8 \%)$ \\
\hline Other & $54(33.3 \%)$ \\
\hline \multicolumn{2}{|l|}{ Pulmonary metastasis } \\
\hline Size [mm] (median) & $10.5[7-16]$ \\
\hline Margins [mm] (median) & $6[3-12]$ \\
\hline RO & $159(98.2 \%)$ \\
\hline R1 & $3(1.9 \%)$ \\
\hline Lymph node involvement & $9(5.6 \%)$ \\
\hline \multicolumn{2}{|l|}{ First PM } \\
\hline VATS & $136(83.9 \%)$ \\
\hline Thoracotomy & $26(16.1 \%)$ \\
\hline Wedge resection & 119 (73.5\%) \\
\hline Segmentectomy & $12(7.4 \%)$ \\
\hline Lobectomy & $29(17.9 \%)$ \\
\hline Pneumonectomy & $2(1.2 \%)$ \\
\hline Mediastinal lymph nodes dissection & $36(22.2 \%)$ \\
\hline \multicolumn{2}{|l|}{ Post-operative outcomes } \\
\hline Overall 30-d mortality & 0 \\
\hline Overall 30-d morbidity & $19(11.7 \%)$ \\
\hline Pulmonary complications & $12(7.4 \%)$ \\
\hline Cardiac complications & $3(1.9 \%)$ \\
\hline Duration of drainage [days] (median) & 1 [IQR 1-2] \\
\hline Duration of hospital stay [days] (median) & 4 [IQR 3-7] \\
\hline Readmission (30-d) & $2(1.2 \%)$ \\
\hline Re-operation (30-d) & $1(0.6 \%)$ \\
\hline
\end{tabular}

PM pulmonary metastasectomy, cardiopathy (defined as the presence of ischemic events in the past, cardiac insufficiency, arrhythmia or aortic aneurysm), high blood pressure (defined as systolic arterial pressure $>140 \mathrm{mmHg}$ ), pulmonary disease (defined as the presence of chronic obstructive pulmonary disease, fibrosis, pulmonary hypertension or sleep apnoea syndrome), diabetes (defined as fasting plasma glucose $>7 \mathrm{mmol} / \mathrm{l}$ ), renal failure (defined as glomerular filtration rate $<30 \mathrm{ml} / \mathrm{min} / 1.73 \mathrm{~m}^{2}$ ). R0 (defined as the absence of cancer cells seen microscopically at the tumor site), R1 (defined as the presence of cancer cells microscopically at the tumor site), R2 (defined as macroscopic residual tumor at cancer site or regional lymph nodes) 
Table 2 Recurrence characteristics

\begin{tabular}{ll}
\hline & $\begin{array}{l}\text { Number } \\
\text { of patients } \\
\text { (percentage) }\end{array}$ \\
\hline Recurrence & $93(57.4 \%)$ \\
Lung only & $21(13 \%)$ \\
Distant & $26(16.1 \%)$ \\
Both lung and distant & $46(28.4 \%)$ \\
Ipsilateral & $34(50.8 \%)$ \\
Controlateral & $21(31.3 \%)$ \\
Bilateral & $12(17.9 \%)$ \\
Repeated pulmonary metastasectomy & $35(21.6 \%)$ \\
VATS & $27(77.1 \%)$ \\
Thoracotomy & $9(25.7 \%)$ \\
Wedge resection & $27(77.1 \%)$ \\
Segmentectomy & $6(17.1 \%)$ \\
Lobectomy & $6(17.1 \%)$ \\
Pneumonectomy & $1(2.9 \%)$ \\
Mediastinal lymph nodes dissection & $8(22.9 \%)$ \\
\hline
\end{tabular}

VATS video-assisted thoracic surgery

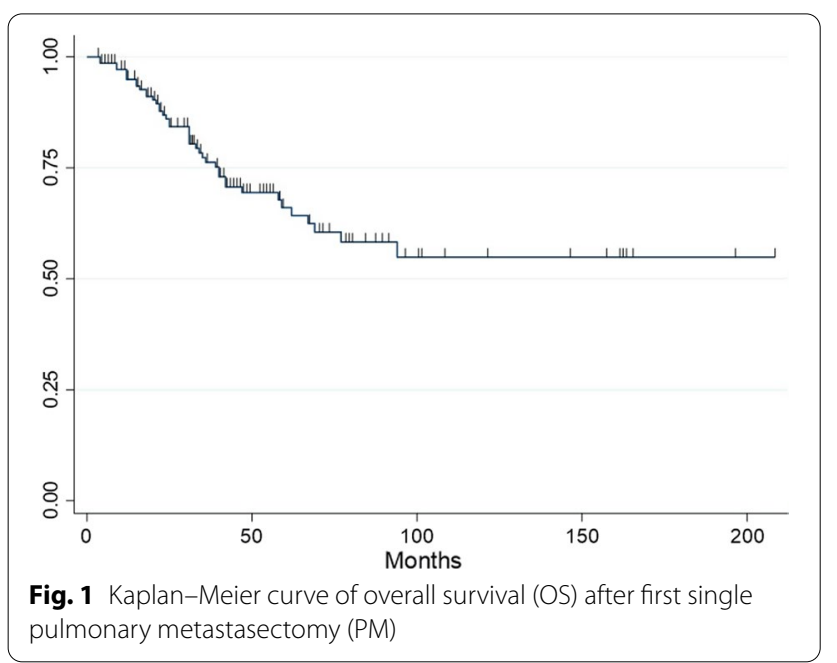

wedge resection in $77.1 \%$ of cases. The 5 -year OS was $67 \%$ after first PM (Fig. 1).

Several prognostic factors of recurrence after PM were identified by uni- and multivariable analysis. The factors found to be significant after multivariable analysis were: age $<70$ years (HR 1.77 , 95\% CI 1.062.96, $p=0.03$ ); non-colorectal tumour (HR 1.84, 95\% CI 1.14-2.96), $p=0.01)$; presence of prior extra-thoracic metastases (HR 1.61, 95\% CI 1.05-2.47, $p=0.03$ ) (Table 3). Similarly, two factors were associated with an increased risk of death: non-colorectal tumour (HR 2.40, 95\% CI 1.11-5.22, $p=0.03$ ) and mediastinal lymph nodes involvement (HR 3.42, 95\% CI 1.03-11.41, $p=0.04$ ) (Table 4).

\section{Discussion}

We report on a large series of 162 patients with a single pulmonary metastasis who underwent a PM from 2003 to 2018. Our data indicates that these patients present favourable overall survival (median 31.5 months; 5 -year OS of $67 \%$ ). However, a majority of patients (57.4\%) developed recurrences with a short median DFI2 (11 months) after PM.

A PM is generally proposed when the following selection criteria are met: a controlled primary tumour, no extra-thoracic or mediastinal lymph node metastatic spread and sufficient pulmonary reserves to tolerate the resection of all identified metastases [2]. The improvement of surgical techniques and radiological imaging and the recent advances in systemic therapies with the development of new chemotherapeutic agents have contributed to an increase in the numbers of PM procedures. Patients with solid tumours frequently present a single pulmonary nodule, which may not necessarily be a metastasis. Indeed, pulmonary metastases may radiologically resemble other conditions, such as primary lung cancers or benign inflammatory lesions. Surgical resection is sometimes the only way to histologically confirm or infirm the diagnosis of a metastatic disease. Interestingly, in a recent series of cancer patients, VATS resection of solitary nodules allowed the diagnosis of metastases in only $50 \%$ of cases [3]. This point is particularly relevant in the context of the development of non-surgical therapies, such as stereotaxic radiotherapy or radiological ablative techniques, where histological diagnosis is rarely reported.

In surgical series, the presence of a solitary metastasis is a frequent situation and represents $47 \%$ to $70 \%$ of all pulmonary metastatic cases $[5,12]$. Many studies have reported that the number of pulmonary metastases is a prognostic factor of survival [5, 13-17]. In colorectal cancer patients, a recent large systematic review including 8361 patients undergoing PM reported that the isolated unilateral lung metastases represented a favourable prognostic factor [12]. A meta-analysis including more than 20 studies showed an increased risk of death (HR 2.04) for multiple lung metastases [9]. In a large series of 615 patients with colorectal cancer, Cho et al. also demonstrated that the number of pulmonary metastases directly influenced the survival, with an overall 5-year survival rate of $70 \%$ in the subgroup with single pulmonary metastases compared to $56.2 \%$ in the subgroup with $2-3$ metastases $(p<0.001)$ [10]. Similar results with better survival for patients with a solitary pulmonary metastasis were also described for other primary tumours: sarcoma 
Table 3 Uni- and multivariate analyses of prognostic factors of recurrence after single pulmonary metastasectomy (PM)

\begin{tabular}{|c|c|c|c|c|}
\hline \multirow[t]{2}{*}{ Variables } & \multicolumn{2}{|l|}{ Univariate } & \multicolumn{2}{|l|}{ Multivariate } \\
\hline & $\mathrm{HR}(95 \% \mathrm{Cl})$ & $p$ value & $\mathrm{HR}(95 \% \mathrm{Cl})$ & $p$ value \\
\hline Female sex & $1.02(0.67-1.55)$ & 0.92 & & \\
\hline Age $<70$ years & $1.84(1.1-3.06)$ & 0.02 & $1.77(1.06-2.96)$ & 0.03 \\
\hline Non-colorectal tumour & $1.76(1.09-2.84)$ & 0.02 & $1.84(1.14-2.96)$ & 0.01 \\
\hline Previous extra-thoracic metastases & $1.65(1.08-2.54)$ & 0.02 & $1.61(1.05-2.47)$ & 0.03 \\
\hline Chemotherapy before first PM & $0.84(0.55-1.28)$ & 0.42 & & \\
\hline DFI1 $<12$ months & $1.29(0.81-2.02)$ & 0.28 & & \\
\hline Synchronous metastasis & $1.42(0.77-2.63)$ & 0.26 & & \\
\hline VATS & $1.15(0.61-2.17)$ & 0.66 & & \\
\hline Wedge resection & $1.26(0.77-2.09)$ & 0.35 & & \\
\hline Margins of the pulmonary metastasis $<5 \mathrm{~mm}$ & $1.13(0.75-1.72)$ & 0.54 & & \\
\hline Size of the pulmonary metastasis $<20 \mathrm{~mm}$ & $0.91(0.53-1.57)$ & 0.74 & & \\
\hline Mediastinal lymph nodes involvement & $1.56(0.57-4.29)$ & 0.39 & & \\
\hline
\end{tabular}

HR hazard ratio, $C l$ confidence interval, $P M$ pulmonary metastasectomy, DFI1 disease-free interval (defined as the interval between primary tumour resection and first PM), VATS Video-Assisted Thoracic Surgery

Table 4 Uni- and multivariate analyses of prognostic factors of worse survival after single pulmonary metastasectomy (PM)

\begin{tabular}{|c|c|c|c|c|}
\hline \multirow[t]{2}{*}{ Variables } & \multicolumn{2}{|l|}{ Univariate } & \multicolumn{2}{|l|}{ Multivariate } \\
\hline & $\mathrm{HR}(95 \% \mathrm{Cl})$ & $p$ value & $\mathrm{HR}(95 \% \mathrm{Cl})$ & $p$ value \\
\hline Female sex & $1.27(0.69-2.34)$ & 0.45 & & \\
\hline Age $>70$ years & $0.83(0.39-1.75)$ & 0.62 & & \\
\hline Non-colorectal tumour & $2.44(1.12-5.29)$ & 0.02 & $2.40(1.11-5.22)$ & 0.03 \\
\hline Previous extra-thoracic metastases & $0.81(0.42-1.56)$ & 0.55 & & \\
\hline Chemotherapy before first PM & $1.00(0.54-1.85)$ & 0.99 & & \\
\hline DFI1 $<12$ months & $1.32(0.66-2.65)$ & 0.43 & & \\
\hline Synchronous metastasis & $1.97(0.82-4.70)$ & 0.13 & & \\
\hline VATS & $1.04(0.44-2.48)$ & 0.93 & & \\
\hline Wedge resection & $0.93(0.47-1.86)$ & 0.84 & & \\
\hline Margins of the pulmonary metastasis $<5 \mathrm{~mm}$ & $0.80(0.43-1.49)$ & 0.48 & & \\
\hline Size of the pulmonary metastasis $<20 \mathrm{~mm}$ & $0.96(0.43-2.18)$ & 0.93 & & \\
\hline Mediastinal lymph nodes involvement & $3.60(1.08-12.03)$ & 0.04 & $3.42(1.03-11.41)$ & 0.04 \\
\hline
\end{tabular}

$H R$ hazard ratio, $C I$ confidence interval, $P M$ pulmonary metastasectomy, $D F / 1$ disease-free interval (defined as the interval between primary tumour resection and first PM), VATS Video-Assisted Thoracic Surgery

(HR 1.16, 95\% CI 1.10-2.503, $p=0.016)$, melanoma (HR 1.4, 95\% CI 1.1-1.7, $p=0.013$ ) and renal cell carcinoma (HR 1.55, 95\% CI 1.18-2.03, $p=0.002$ ) [13, 18, 19].

In our study, we decided to focus on the population of patients bearing one pulmonary metastasis only because this population represents a non-negligible fraction of all pulmonary metastatic patients, and because it is by definition most amenable to surgical cure of its disease. Our aim was to evaluate risk factors for recurrence and risk factors for worse survival specifically for this population. Most of the PM were performed by VATS (84\%). Pulmonary metastases are generally peripheral and small-sized (median diameter: $10 \mathrm{~mm}$ ), making them easily accessible for a non-anatomical resection by VATS. However, some surgeons still perform thoracotomies in order to palpate the lung and identify other lesions. Nowadays, this paradigm is changing for solitary metastases and VATS approach is becoming the preferred approach, as showed in a survey of cardiothoracic surgeons in Great Britain and Ireland reporting that VATS was used by $85 \%$ of surgeons in case of isolated pulmonary metastatic lesions [20]. Indeed, the 1-mm thin-slice CT-scans are very sensitive and can detect nodules of less than $5 \mathrm{~mm}$ in diameter, making the bimanual palpation obsolete.

The concordance between radiological imaging and pathological findings was analyzed by the Spanish 
prospective registry of PM [21]. In this study, solitary nodules were present in $73 \%$ of colorectal cancer patients who underwent thoracotomy with bimanual palpation of the lung. The radiological and pathological agreement was $95 \%$. In another series, only $7 \%$ of patients with single nodule on pre-operative CT-scan presented more metastatic lesions on pathological analysis after resection by thoracotomy [22]. Thus, these recent results suggest that VATS is a valid approach, at least for patients with a single lesion on pre-operative imaging. In our study, the Cox regression analysis did not find any correlation between the surgical approach (VATS vs. thoracotomy) and the increased risk of recurrence (HR 1.15, 95\% CI 0.61-2.17, $p=0.66$ ).

Wedge resections using staplers accounted for $74.1 \%$ of cases. Anatomical resections (segmentectomy or lobectomy) were reserved for centrally located or larger lesions, the objective being to achieve safe margins, a result known to improve prognosis [23]. We did not find any association between the type of surgical resection and the survival prognosis (HR 0.93, 95\% CI $0.44-2.48, p=0.93$ ). Major resections could be justified for selected patients with larger or centrally located pulmonary metastases with favorable results, as demonstrated in a multicenter prospective study reporting an increased survival rate in comparison with non-anatomical resections for colorectal cancer patients with pulmonary metastases (55 vs. 28.3 months) [24].

The post-operative outcomes were favourable with an overall low morbidity of $11.7 \%$ with minor complications and a 30 -day mortality rate of $0 \%$. These results are consistent with other surgical series about pulmonary metastases $[25,26]$.

Despite our expectation to observe better prognosis for patients bearing solitary nodules, we observed a recurrence rate of $57.4 \%$ in that population. We identified three prognostic factors of recurrence: age under 70 years (HR 1.77, 95\% CI 1.06-2.96, $p=0.03$ ), prior treatment of extra-thoracic metastases (HR 1.61, 95\% CI 1.05-2.47, $p=0.03$ ) and non-colorectal origin (HR 1.84, 95\% CI 1.14-2.96, $p=0.01$ ). These elements are generally correlated with a biologically aggressive behaviour of the primary tumour, which are more prone to generating recurrences.

In our study, of the $93(57.4 \%)$ patients who presented a recurrence, only $35(21.6 \%)$ fulfilled the criteria to undergo an RPM, due to the invasion of other organs or poor residual lung capacity. Our indications for redo surgery are identical to the indications for initial surgery. Interestingly, most of the RPM have been performed by VATS $(77.1 \%)$ and by wedge resection (77.1\%). We observed that VATS procedures induced fewer adhesions and chest wall sequelae than thoracotomies. Thus, repeated VATS procedures were easier to perform.

The 5-year overall survival rate of single PM was $67 \%$, which compares favourably with data from recent literature. With our results, we could identify two factors predictive of shortened survival: non-colorectal tumour origin (HR 2.40, 95\% CI 1.11-5.22, $p=0.03$ ) and mediastinal lymph nodes involvement (HR 3.42, 95\% CI 1.03$11.41, p=0.04)$. The primary tumour origin has been shown to influence survival with better survival rates in epithelial cancers than in sarcomas or melanomas [5]. Hirai et al. showed that colorectal cancer patients had a better survival rate than patients with other primary organs involved $(p=0.003)$ [4]. In our study, we chose to analyse only two subgroups of primary tumours (colorectal and non-colorectal) because of the high frequency of colorectal tumours (31.5\%) compared to other types. The non-colorectal subgroup included melanoma, sarcoma, and others (germ cell, head and neck, breast, urological, gynaecological, thyroid and other).

Many studies have reported a long DFI as being a favourable prognostic factor of survival [5, 13]. In a recent meta-analysis of renal cancer patients with pulmonary metastases, both the synchronous metastases and a short DFI were associated with poor survival rates [13]. In our study, a DFI1 $<12$ months did not have any correlation with prognosis.

Lymph node dissection was realized in $22.2 \%$ of cases and only nine patients $(5.6 \%)$ presented hilar or mediastinal lymph node involvement, which was correlated to worse survival rate (HR 3.42, 95\% CI 1.03-11.41, $p=0.04$ ). While survival of pulmonary metastatic patients is affected by metastatic invasion of the lymph nodes, it remains unclear if systematic lymph node dissection during solitary PM brings any benefits in terms of local recurrence or survival [1]. Lymph node dissection was not performed routinely for solitary peripheral lesions and was reserved for centrally located or larger lesions requiring an anatomical resection. Our rate of lymph node dissection is relatively low in comparison with other series, but does not seem to correlate to the survival rate in this group of patients and in the timeframe that we studied.

Our study presents several limitations, the first one being the retrospective single-center design with a small collective of patients. Next, our study included only patients who underwent a surgical resection of their single pulmonary metastasis. Other patients with a single pulmonary metastasis who were not treated by surgery were not included, thus creating a selection bias. However, our selection criteria mentioned this factor and 5-year survival and other outcomes were described only for included patients, namely those who underwent 
surgery. Another limitation is the variety of primary tumor types, thus inducing a heterogeneity of the studied population. However, we selected only the patients with a single pulmonary metastasis and reported the primary tumor types, which we stratified along clear lines. Moreover, the patients were included over a 15-year period, thus smoothing out influences on the prognosis that might be due to the evolution of systemic and radiologic therapies. It should be noted also that the exact 5-year survival figures could only be calculated on a fraction of the patient population (those treated until 2015).

\section{Conclusions}

In conclusion, our results demonstrate that surgical resection of a single pulmonary metastasis is beneficial for the patients, thanks to low post-operative morbidity and mortality rates, as well as to an acceptable survival duration. The VATS approach should be preferred when feasible, owing to its low morbidity and mortality rates. However, local or distant recurrences are frequent and RPM can be achieved in a substantial number of cases, especially when the initial PM was carried out by VATS. We found risk factors for recurrence to be a younger age $(<70)$, primary tumour of non-colorectal origin and a history of extra-thoracic metastases. Similarly, risk factors for worse survival were found to be a primary tumour of non-colorectal origin and invasion of the mediastinal lymph nodes.

\begin{abstract}
Abbreviations
PM: Pulmonary metastasectomy; VATS: Video-assisted thoracic surgery; HR: Hazard ratio; PET: Positron emission tomography; FDG: Fluorodeoxy-glucose; DFI1: Disease-free interval 1 (defined as the interval between primary tumour resection and first PM); DFI2: Disease-free interval 2 (defined as the interval between first PM and cancer recurrence); OS: Overall survival; IQR: Interquartile range; RPM: Repeated pulmonary metastasectomy; Cl: Confidence interval.
\end{abstract}

\section{Acknowledgements}

Not applicable.

\section{Authors' contributions}

CF and MG collected, analyzed and interpreted the patient data. CF, MG and $\mathrm{MZ}$ performed the statistical analysis. All authors read and approved the final manuscript.

\section{Funding}

No funding.

\section{Availability of data and materials}

The datasets used and/or analyzed during the current study are available from the corresponding author on reasonable request.

\section{Declarations}

Ethics approval and consent to participate

The Local Ethics Committee (CER-VD, Avenue de Chailly 23, 1012 Lausanne, VD Switzerland, Secretariat.CER@vd.ch) approved the study (No. 2019-02474) and individual consent was waived.

\section{Consent for publication}

Individual consent was waived.

\section{Competing interests}

The authors declare that they have no competing interests.

\section{Author details}

${ }^{1}$ Service of Thoracic Surgery, Lausanne University Hospital (CHUV), Rue du Bugnon 46, 1011 Lausanne, Switzerland. ${ }^{2}$ Faculty of Biology and Medicine, University of Lausanne (UNIL), Rue du Bugnon 21, 1011 Lausanne, Switzerland.

Received: 22 December 2020 Accepted: 19 December 2021

Published online: 28 December 2021

\section{References}

1. Gonzalez M, Zellweger M, Nardini M, et al. Precision surgery in lung metastasectomy. Future Oncol. 2019. https://doi.org/10.2217/ fon-2018-0713.

2. Thomford NR, Woolner LB, Clagett OT. The surgical treatment of metastatic tumors in the lungs. J Thorac Cardiovasc Surg. 1965;49:357-63.

3. Bellier J, Perentes JY, Abdelnour-Berchtold E, et al. A plea for thoracoscopic resection of solitary pulmonary nodule in cancer patients. Surg Endosc. 2017;31:4705-10

4. Hirai F, Kinoshita I, Matsubara T, et al. Which primary organ is most suitable for performing pulmonary metastasectomy? Anticancer Res. 2018:38:1041-5.

5. Pastorino $U$, Buyse $M$, Friedel $G$, et al. Long-term results of lung metastasectomy: prognostic analyses based on 5206 cases. J Thorac Cardiovasc Surg. 1997;113:37-49.

6. De Baère T, Aupérin A, Deschamps F, et al. Radiofrequency ablation is a valid treatment option for lung metastases: experience in 566 patients with 1037 metastases. Ann Oncol. 2015;26:987-91.

7. Meng D, Fu L, Wang L, et al. Video-assisted thoracoscopic surgery versus open thoracotomy in pulmonary metastasectomy: a meta-analysis of observational studies. Interact Cardiovasc Thorac Surg. 2016;22:200-6.

8. Higashiyama M, Tokunaga T, Nakagiri T, et al. Pulmonary metastasectomy: outcomes and issues according to the type of surgical resection. Gen Thorac Cardiovasc Surg. 2015;63:320-30.

9. Gonzalez M, Poncet A, Combescure C, et al. Risk factors for survival after lung metastasectomy in colorectal cancer patients: a systematic review and meta-analysis. Ann Surg Oncol. 2013;20:572-9.

10. Cho JH, Kim S, Namgung M, et al. The prognostic importance of the number of metastases in pulmonary metastasectomy of colorectal cancer. World J Surg Oncol. 2015;13:222.

11. Zellweger M, Abdelnour-Berchtold E, Krueger T, et al. Surgical treatment of pulmonary metastasis in colorectal cancer patients: current practice and results. Crit Rev Oncol Hematol. 2018;127:105-16.

12. Abdelnour-Berchtold E, Perentes JY, Ris H-B, et al. Survival and local recurrence after video-assisted thoracoscopic lung metastasectomy. World J Surg. 2016:40:373-9.

13. Zhao Y, Li J, Li C, et al. Prognostic factors for overall survival after lung metastasectomy in renal cell cancer patients: a systematic review and meta-analysis. Int J Surg. 2017:41:70-7.

14. Zabaleta J, lida T, Falcoz PE, et al. Individual data meta-analysis for the study of survival after pulmonary metastasectomy in colorectal cancer patients: a history of resected liver metastases worsens the prognosis. Eur J Surg Oncol. 2018;44:1006-12.

15. Kim JY, Park IJ, Kim HR, et al. Post-pulmonary metastasectomy prognosis after curative resection for colorectal cancer. Oncotarget. 2017:8:36566-77.

16. Blackmon SH, Stephens EH, Correa AM, et al. Predictors of recurrent pulmonary metastases and survival after pulmonary metastasectomy for colorectal cancer. Ann Thorac Surg. 2012;94:1802-9.

17. Inoue $M$, Ohta M, luchi $K$, et al. Benefits of surgery for patients with pulmonary metastases from colorectal carcinoma. Ann Thorac Surg. 2004;78:238-44.

18. Mizuno T, Taniguchi T, Ishikawa Y, et al. Pulmonary metastasectomy for osteogenic and soft tissue sarcoma: who really benefits from surgical treatment? Eur J Cardiothorac Surg. 2013;43:795-9. 
19. Chua TC, Scolyer RA, Kennedy CW, et al. Surgical management of melanoma lung metastasis: an analysis of survival outcomes in 292 consecutive patients. Ann Surg Oncol. 2012;19:1774-81.

20. Jegatheeswaran S, Satyadas T, Sheen AJ, et al. Thoracic surgical management of colorectal lung metastases: a questionnaire survey of members of the Society for Cardiothoracic Surgery in Great Britain and Ireland. Ann R Coll Surg Engl. 2013;95:140-3.

21. Marron MC, Lora D, Gamez P, et al. Agreement between computed tomography and pathologic nodule counts in colorectal lung metastases. Ann Thorac Surg. 2016;101:259-65.

22. Ludwig C, Cerinza J, Passlick B, Stoelben E. Comparison of the number of pre-, intra- and post-operative lung metastases. Eur J Cardiothorac Surg. 2008:33:470-2.

23. Nelson DB, Tayob N, Mitchell KG, et al. Surgical margins and risk of local recurrence after wedge resection of colorectal pulmonary metastases. J Thorac Cardiovasc Surg. 2019;157:1648-55.

24. Hernandez J, Molins L, Fibla JJ, et al. Role of major resection in pulmonary metastasectomy for colorectal cancer in the Spanish prospective multicenter study (GECMP-CCR). Ann Oncol. 2016;27:850-5.

25. Rodriguez-Fuster A, Belda-Sanchis J, Aguilo R, et al. Morbidity and mortality in a large series of surgical patients with pulmonary metastases of colorectal carcinoma: a prospective multicentre Spanish study (GECMPCCR-SEPAR). Eur J Cardiothorac Surg. 2014;45:671-6.

26. Menna C, Berardi G, Tierno SM, et al. Do repeated operations for recurrent colorectal lung metastases result in improved survival? Ann Thorac Surg. 2018;106:421-7.

\section{Publisher's Note}

Springer Nature remains neutral with regard to jurisdictional claims in published maps and institutional affiliations.

- fast, convenient online submission

- thorough peer review by experienced researchers in your field

- rapid publication on acceptance

- support for research data, including large and complex data types

- gold Open Access which fosters wider collaboration and increased citations

- maximum visibility for your research: over $100 \mathrm{M}$ website views per year

At BMC, research is always in progress.

Learn more biomedcentral.com/submissions 